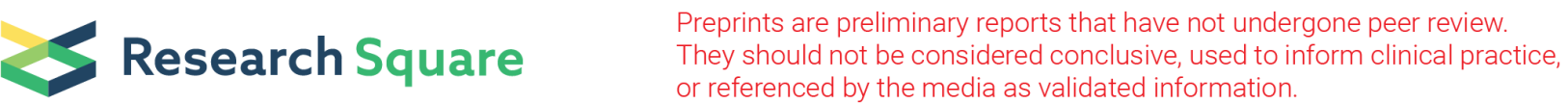

\section{Quantitative Study on the Efficacy of Acupuncture in the Treatment of Menopausal Hot Flashes and Its Comparison with Non-Hormonal Drugs}

\section{Ting Li}

Shanghai University of Traditional Chinese Medicine https://orcid.org/0000-0002-6229-7781

\section{Yi Zhang}

Shanghai University of Traditional Chinese Medicine

Qingqing Cheng

Shanghai University of Traditional Chinese Medicine

Mengyuan Hou

Shanghai University of Traditional Chinese Medicine

Xijun Zheng

Shanghai University of Traditional Chinese Medicine

Qingshan Zheng

Shanghai University of Traditional Chinese Medicine

Lujin Li ( $\nabla$ lilujin666@163.com )

Shanghai University of Traditional Chinese Medicine

Research

Keywords: Hot flashes, Acupuncture, Sham acupuncture, Placebo, Non-hormonal drugs

Posted Date: October 16th, 2020

DOl: https://doi.org/10.21203/rs.3.rs-90756/v1

License: (9) This work is licensed under a Creative Commons Attribution 4.0 International License.

Read Full License 


\section{Abstract}

Background The efficacy of acupuncture in the treatment of menopausal hot flashes has always been controversial, which limited the use of acupuncture. This study aimed to compare the efficacy of acupuncture to that of sham acupuncture (SA), placebo pills, and non-hormonal drugs to provide the necessary quantitative information for establishing medication guidelines for menopausal hot flashes.

Methods A comprehensive literature search was performed using public databases. Randomized clinical studies on acupuncture therapy for the treatment of hot flashes in menopausal women were identified. A time-course model was established to describe the efficacy characteristics of acupuncture and SA, which were compared with the efficacy of non-hormonal drugs and placebo pills reported in the literature.

Results A total of 17 studies involving 1123 subjects were included. It was found that the baseline number of hot flashes was an important factor affecting the efficacy of acupuncture and SA. After correcting the baseline to eight hot flashes per day, the frequency of hot flashes decreased from baseline for traditional acupuncture (TA), electro-acupuncture (EA), TA\&EA (merger analysis of TA and EA), and SA were 3.1 (95\% Cl: 2.8, 3.4), 3.6 (95\% Cl: 3.2, 4.0), 3.2 (95\% Cl: 2.9, 3.5), and 2.6 (95\% Cl: $2.2,3.0)$ times/day at week 8 , respectively. Compared with findings reported in the literature, we found the efficacy of EA was comparable to that of selective serotonin reuptake inhibitors/serotonin-norepinephrine reuptake inhibitors (SSRIs/SNRIs) and neuroleptic agents such as gabapentin and escitalopram. Furthermore, the efficacy of TA\&EA was significantly higher than that of placebo pills $(2.3,95 \% \mathrm{Cl}: 1.8,2.9)$.

Conclusions The efficacy of TA\&EA was higher than that of SA and significantly higher than that of placebo pills. The efficacy of EA was higher than that of TA, significantly higher than that of SA, and comparable to that of SSRIs/SNRIs and neuroleptic agents.

\section{Background}

Hot flashes are disturbing vasomotor symptoms seen during menopause that are caused by low estrogen levels, always accompanied by a large amount of sweating and a sensation of intense heat in the face, neck, or chest [1]. In America, approximately $75 \%$ of menopausal women experience hot flashes, which may profoundly impact their quality of life [2]. Hormone replacement therapy (HRT) can significantly alleviate menopausal hot flashes, however, due to safety concerns, HRT products are contraindicated for women who have breast cancer or a history of breast cancer, venous thromboembolism or a history of venous thromboembolism, thrombophilia, or a history of stroke or myocardial infarction [3].

The selective serotonin reuptake inhibitor (SSRI) paroxetine was approved by the US Food and Drug Administration (FDA) in 2013 and was the first non-hormonal drug approved for the treatment of menopausal hot flashes [4]. However, the efficacy of paroxetine in relieving menopausal hot flashes is not obvious. The decreased frequency of hot flashes after 12 weeks of treatment is only $14.6 \%$ higher than that of the placebo, which is equivalent to only $40 \%$ of the efficacy of estradiol [5]. Other non-hormonal drugs such as gabapentin, clonidine, SSRIs (except paroxetine), and serotonin norepinephrine reuptake 


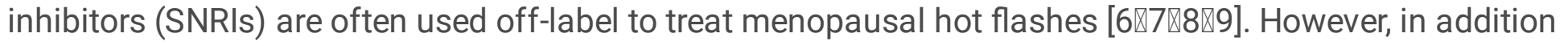
to a wide range of central side effects and the risk of drug interactions [10], the efficacies of these drugs fail to demonstrate much of an advantage and their maximum efficacy is no more than half of that of estradiol [5].

Phytoestrogens are also widely used in the treatment of menopausal hot flashes because of their estrogen-like effects [11]. However, their actual efficacy remains controversial [12]. For instance, our previous study suggested that the maximum efficacy of soy isoflavones was higher than that of paroxetine, but the onset of action was very slow and at least 16 weeks of treatment was needed to reach or surpass the efficacy of paroxetine [5]. As the efficacy of non-hormone drugs is generally poor, some individuals use complementary or alternative therapies of which acupuncture is one of the most common [13]. The prevalence of acupuncture use by women to treat menopausal hot flashes is estimated to be 1$10.4 \%$ [1]. A large number of studies have confirmed that acupuncture can promote the release of endogenous analgesics, thereby preventing the development of chronic pain [14]. However, the mechanism of acupuncture in alleviating menopausal hot flashes remains unclear [15囚16]. A randomized controlled trial published in 2016 showed that acupuncture has no improved efficacy in alleviating menopausal hot flashes compared to that of non-intrusive sham acupuncture (SA) [17]. Many researchers believe that acupuncture is only a powerful placebo and that its efficacy comes primarily from strong patient beliefs and expectations regarding acupuncture, rather than any direct "biological effect" on the body. Opponents argue that non-intrusive SA may also produce certain biological effects due to skin irritation that are not completely ineffective [18]. Therefore, using non-intrusive SA as a control may actually improve the placebo effect, making it difficult to formulate accurate conclusions in acupuncture trials [1].

Although there have been a number of meta-analyses on the efficacy of acupuncture in relieving

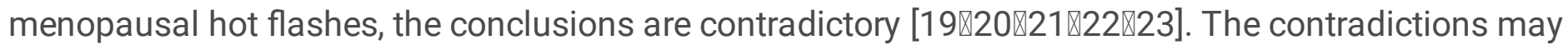
result from limitations of the studies. First, most acupuncture trials report repeated measurements of hot flashes over time. However, previous meta-analyses often aggregated the results of only the final time point (ranging from 4 to 24 weeks), which not only excludes valuable information from other follow-up time points, but also results in bias estimates of efficacy due to different follow-up time points being used [11]. Second, there is a large amount of heterogeneity between the studies [20], such as treatment duration, baseline frequency of hot flashes, the method of blinding, and other factors that affect the reliability of the conclusions. Model-based meta-analysis (MBMA) is an efficient type of meta-analysis that explicitly incorporates the effects of treatment duration and other covariates using pharmacology models, thereby reducing the heterogeneity among studies. MBMA has become an important analytical method in model-informed drug development (MIDD) [5ष11].

We previously determined the distribution of efficacy for placebos and non-hormonal drugs in relieving menopausal hot flashes using MBMA [4ه5]. In the current study, we used MBMA to quantitatively analyze the efficacy characteristics of acupuncture in treating menopausal hot flashes. This study focused on comparing the efficacy of acupuncture with that of SA, the efficacy of SA with that of an oral 
pharmacological placebo, and the efficacy of acupuncture with that of non-hormonal drugs in order to provide the quantitative information necessary for establishing medication guidelines for treating menopausal hot flashes.

\section{Methods}

\section{Search strategy}

We carried out a comprehensive search for literature indexed in PubMed, EMBASE, Cochrane Library, Web of Science, and SCOPUS databases with publication dates ranging from the date of inception of each database until January 8, 2019. The search terms included "hot flashes" and "acupuncture". Only clinical trials were included and the language was restricted to English. The detailed search strategies are described in the Supplementary Search Strategy.

\section{Inclusion and exclusion criteria}

Only studies that met the following criteria were included: (1) randomized controlled acupuncture trials, (2) subjects were pre-menopausal and post-menopausal women or patients with a history of breast cancer, (3) studies reporting the frequency of hot flashes at baseline and after treatment, and (4) if the study was a cross-over design, only data from the first period were included in order to avoid the legacy effect. Any cross-over trials that failed to report outcomes in the first stage were excluded. To reduce heterogeneity, participants with other comorbidities or interventions of acupuncture combined with other treatments were excluded.

\section{Data extraction}

Data were independently extracted by two researchers and checked by a third person for any discrepancies. A Microsoft Excel (version 16.0.12130.20232) database was used to categorize relevant information from the included studies. The information collected included literature characteristics (author, year of publication, and clinical trial registration number), trial design (grouping, sample size, treatment duration, blinding method, frequency of acupuncture treatment, and total acupuncture times), characteristics of participants (age, weight, region, frequency of hot flashes at baseline, duration of disease, and type of subjects), and clinical outcomes (frequency of hot flashes for each follow-up time point).

Data from intention-to-treat (ITT) groups were entered when available. For published per-protocol (PP) data, unreported sample sizes during treatment were calculated based on the endpoint sample size. If efficacy results were presented in graphs, the digitizing software Engauge Digitizer (version 4.1, 2002, by Mark Mitchell) was used to extract the graphical data. Data extraction errors between two independent 
researchers were not allowed to exceed $2 \%$. If the error was greater than $2 \%$, data extraction was repeated and the mean values were considered as the final results.

\section{Risk of bias assessment}

Two investigators independently extracted relevant information and assessed the risk of bias using the Cochrane Risk of Bias Tool [24]. Any disagreement was resolved through discussion with a third investigator. The evaluation items included random sequence generation, allocation concealment, blinding of participants and personnel, blinding for outcome assessment, incomplete outcome data, selective reporting, and other biases. Other biases were defined as trials in which baseline characteristics were not comparable between different treatment groups.

These studies were graded as high, moderate, or low quality based on the following criteria: (1) studies were considered to be of high quality when both randomization and allocation concealment were assessed as low risks of bias, and all other items were assessed as low or unclear risk of bias in a trial; (2) studies were considered to be of low quality if either randomization or allocation concealment was assessed as a high risk of bias, regardless of the risk of other items; (3) studies were considered to be of moderate quality if they did not meet the criteria for high or low quality.

\section{Model building}

In a previous study, we found that changes in the frequency of hot flashes varied with time and reached a plateau, which is consistent with the $\mathrm{E}_{\max }$ model. The $\mathrm{E}_{\max }$ model demonstrates good biological plausibility and is frequently used for modeling pharmacodynamic properties of drugs [25]. This model has two important parameters, $\mathrm{E}_{\max }$ and $\mathrm{ET}_{50}$. $\mathrm{E}_{\max }$ is the maximum possible efficacy that treatment can achieve. $\mathrm{ET}_{50}$ is the time point at which $50 \%$ of the maximum efficacy has been achieved, which represents the speed of the onset of treatment. The combinations of different $E_{\max }$ and $\mathrm{ET}_{50}$ values resulted in different time-course curves. In the current study, the $E_{\max }$ model was used to fit the longitudinal efficacy data of each treatment group with the $\mathrm{E}_{\max }$ and $\mathrm{ET}_{50}$ values of each treatment group being obtained by Bayesian feedback.

Heterogeneity among studies led to large differences in $\mathrm{E}_{\mathrm{max}}$ and $\mathrm{ET}_{50}$ values for different treatment groups. However, some of the differences could be explained by covariates. In the current study, factors that would potentially affect model parameters, such as age, weight, baseline frequency of hot flashes, duration of disease, blinding method, type of subjects, and number of acupunctures per week, were screened as covariates on parameters for both $\mathrm{E}_{\max }$ and $\mathrm{ET}_{50}$. A detailed description of the construction of the model is shown in Supplementary.

\section{Model evaluation}


The goodness-of-fit of the final model was evaluated using diagnostic graphs. The sensitivity of the final model was evaluated using the leave-one-out cross-validation (LOOCV) method [26]. Briefly, data from one trial were sequentially dropped from the full data set and the remaining data were used to fit the final model. The parameter estimates obtained from each data set were compared to investigate the stability of the model. The final model was further validated by a visual predictive check (VPC) [27], which is commonly used to determine if a model is able to reproduce the variability and main trend of observed data. Typically, 1000 datasets were modeled using Monte Carlo simulations based on the final model parameters. The observed data were then compared with the 2.5th, 50th, and 97.5th percentiles of the simulated data to assess the predictive capacity of the final model.

\section{Typical efficacies analysis}

When a covariate was found to have a significant impact on the model parameters, the parameters were corrected for the covariate to ensure that the parameters were comparable at different covariate levels. The methodology for covariate correction is detailed in the Supplementary. A single-arm meta-analysis with random-effects model was then used to summarize the model parameters according to each type of treatment. The typical value and $95 \%$ confidence interval $(\mathrm{Cl})$ of the parameters of each treatment were obtained from the analysis. Based on these parameters, typical efficacy and $95 \% \mathrm{Cl}$ of each treatment at different time points were simulated 1000 times using Monte Carlo Simulation.

\section{Software}

Model parameter estimation was performed using NONMEM 7.4.1 (level 1.0, ICON Development Solutions, New York, NY, USA). Meta-analysis was performed using StataCorp (2013. Stata Statiscal Software: Release 13. College Station, TX, USA: StataCorp LP). Plotting, model simulation, and statistical analysis were performed using R software (version 3.6.0, The R Foundation of Statistical Computing, Vienna, Austria).

\section{Results}

\section{Characteristics of the included studies}

A total of 17 studies including 1123 subjects were ultimately included for analysis (Fig. 1). Of these, seven studies (329 subjects) involved patients with a history of breast cancer. There were three types of treatment involved, including traditional acupuncture $(T A, N=631)$, electro-acupuncture $(E A, N=69)$ and sham acupuncture ( $S A, N=423)$. The mean age of the participants ranged from 50.4 to 61 years with a median age of 54 years. The mean frequency of hot flashes at baseline ranged from 1.9 to 12.9 times per day with a median frequency of 8.4 times per day. The acupuncture frequency ranged from 0.7 to 12 times per week with a median frequency of 1.3 times per week. The treatment duration ranged from 4 to 
24 weeks with a median time of 8 weeks. The sample size of each treatment arm ranged from 10 to 170 with a median value of 24 (Table 1).

Table 1

Brief characteristics of included studies, median(min-max).

\begin{tabular}{|c|c|c|c|c|}
\hline & Overall & $\begin{array}{l}\text { Traditional } \\
\text { acupuncture } \\
\text { (TA) }\end{array}$ & $\begin{array}{l}\text { Electro- } \\
\text { acupuncture } \\
\text { (EA) }\end{array}$ & $\begin{array}{l}\text { Sham } \\
\text { acupuncture } \\
\text { (SA) }\end{array}$ \\
\hline Number of arms & 30 & 13 & 4 & 13 \\
\hline Total sample size & 1123 & 631 & 69 & 423 \\
\hline Sample size per arm & $\begin{array}{l}24(10- \\
170)\end{array}$ & $27(10-170)$ & $17(12-23)$ & $24(10-164)$ \\
\hline Treatment duration, week & $8(4-24)$ & $8(4-24)$ & $12(8-12)$ & $8(4-24)$ \\
\hline Age, years & $\begin{array}{l}54.2(50.4- \\
61)\end{array}$ & $54.7(50.4-61)$ & $\begin{array}{l}53.7(51.2- \\
61)\end{array}$ & $\begin{array}{l}54.3(53- \\
56.5)\end{array}$ \\
\hline $\begin{array}{l}\text { Mean frequency of hot flashes at } \\
\text { baseline, per day }\end{array}$ & $\begin{array}{l}8.5(1.9- \\
12.9)\end{array}$ & $8.7(1.9-12.9)$ & $8.0(6.3-9.6)$ & $\begin{array}{l}8.1(2.2- \\
12.3)\end{array}$ \\
\hline $\begin{array}{l}\text { Type of subjects, } \\
\text { menopause/breast cancer }\end{array}$ & $19 / 11$ & $8 / 5$ & $2 / 2$ & $9 / 4$ \\
\hline
\end{tabular}

Detailed information regarding the included studies is listed in Supplementary Table S1. Of the 17 studies, $3(17.6 \%)$ were high quality, 14 (82.4\%) were medium quality, and no studies were low quality. The quality of the 17 studies is shown in Supplementary Figure S2.

\section{Model establishment and assessment}

The time course of changes in hot flash frequency from baseline was well described by the $E_{\max }$ model. The goodness-of-fit plots of the acupuncture response model indicated a relatively good fit to the observed data (Supplementary Figures S3 and S4). Specifically, plots of population prediction (PRED) vs. observation (OBS) and plots of individual prediction (IPRED) vs. OBS were symmetrically distributed and close to the identity line, indicating good predictions. The plots of time vs. conditional weighted residual errors (CWRES) and plots of PRED vs. CWRES showed no trend and were randomly scattered around the identity line at CWRES $=0$, indicating the suitability of the error model for this study. The VPC plots indicated that the $95 \% \mathrm{Cl}$ of model prediction covered almost all of the observed data, demonstrating good predictability by the model (Fig. 2). The results of LOOCV showed that the distribution of model parameters was stable and only slightly affected by individual trials (Supplementary Figure S5). 
The covariate screening process revealed that the baseline frequency of hot flashes had a significant impact on the $E_{\max }$ value. The final model was expressed as follows:

$$
E_{\max , i}=3.64+(\text { Baseline }-8) \times 0.343+\text { Normal }\left(0,1.049^{2}\right) \quad \text { Equation } 1
$$

$$
E T_{50, i}=1.89
$$

Equation 2

In Eq. $1, E_{m a x, i}$ was the $E_{\max }$ value for the $i^{\text {th }}$ group with 3.64 being the typical $E_{\max }$ value for the overall group and Baseline $e_{i}$ was the frequency of hot flashes for the $\mathrm{i}^{\text {th }}$ group at baseline. For every increase in the number of hot flashes at baseline, the $E_{\max }$ value increased 0.343 times. Therefore, the correction coefficient of the baseline for $E_{\max }$ was 0.343 . Normal $\left(0,1.049^{2}\right)$ was the inter-group variation of the $E_{m a x}$ value using normal distribution with a mean of 0 and variance of 1.049 . The optimal $E_{\text {max,i }}$ values and the standard errors for each group as estimated by Bayesian feedback are shown in Supplementary Table S2. During the model building process, the inter-group variability of $\mathrm{ET}_{50}$ was close to zero, which means that the $\mathrm{ET}_{50}$ value of all the groups was close to 1.89 weeks. Finally, the inter-group variability of $\mathrm{ET}_{50}$ was fixed to 0 to enhance model stability in the final model (Eq. 2).

\section{Typical efficacy analysis}

As the frequency of hot flashes at baseline had a significant impact on the $E_{\max }$ value, it was necessary to perform baseline correction of the $E_{\max }$ value for each treatment group. The correction equation used was as follows:

\section{$E_{\text {max }, i, \text { corrected }}=E_{\max , i}-($ Baselinei -8$) \times 0.374 \quad$ Equation 3}

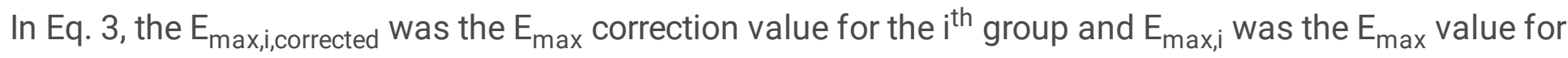
the $i^{\text {th }}$ group. This equation corrected the $E_{\max }$ values at different baselines to a level of 8 , thereby eliminating the impact of the baseline frequency of hot flashes on $E_{\max }$ values when comparing the efficacy characteristics of the different treatment groups.

To compare the efficacy characteristics of different interventions, we summarized the $\mathrm{E}_{\max }$ correction value for each group according to the intervention and estimated the $95 \% \mathrm{Cl}$ of the efficacy at week 8 for each type of intervention. The results revealed that the corrected $E_{\max }$ values of TA\&EA and SA were 4.0 (95\% Cl: $3.6,4.3)$ and 3.2 (95\% Cl: $2.7,3.7)$, respectively. Further division of acupuncture into EA and TA resulted in corrected $\mathrm{E}_{\max }$ values of $4.4(95 \% \mathrm{Cl}: 3.9,4.9)$ and $3.8(95 \% \mathrm{Cl}: 3.5,4.2)$, respectively (Table 2). 
Table 2

Parameter estimations of different intervention

\begin{tabular}{|c|c|c|c|c|c|}
\hline Intervention & $\begin{array}{l}\text { Arms } \\
\text { (sample size) }\end{array}$ & $\begin{array}{l}\mathrm{E}_{\max } \\
(95 \% \mathrm{Cl})\end{array}$ & $\begin{array}{l}E_{\text {max,corrected }} \\
(95 \% \mathrm{Cl})\end{array}$ & $\begin{array}{l}\mathrm{ET}_{50} \\
(95 \% \mathrm{Cl})\end{array}$ & $\begin{array}{l}\text { Corrected efficacy } \\
\text { at week } 8(95 \% \mathrm{Cl})\end{array}$ \\
\hline TA & $13(631)$ & $3.8(3.3,4.4)$ & $3.8(3.5,4.2)$ & $1.9(1.8,2.0)$ & $3.1(2.8,3.4)$ \\
\hline EA & $4(69)$ & $4.4(3.5,5.3)$ & $4.4(3.9,4.9)$ & $1.9(1.8,2.0)$ & $3.6(3.2,4.0)$ \\
\hline TA\&EA & 17(700) & $4.0(3.5,4.4)$ & $4.0(3.6,4.3)$ & $1.9(1.8,2.0)$ & $3.2(2.9,3.5)$ \\
\hline SA & $13(423)$ & $3.1(2.3,3.9)$ & $3.2(2.7,3.7)$ & $1.9(1.8,2.0)$ & $2.6(2.2,3.0)$ \\
\hline Placebo pill $\triangle$ & 11(2191) & $2.7(2.1,3.3)$ & $2.7(2.1,3.3)$ & $1.2(0.8,1.6)$ & $2.3(1.8,2.9)$ \\
\hline \multicolumn{6}{|c|}{$\begin{array}{l}\text { TA: traditional acupuncture, EA: electro-acupuncture, TA\&EA: merger analysis of traditional } \\
\text { acupuncture and electro-acupuncture, SA: sham acupuncture. }\end{array}$} \\
\hline \multicolumn{6}{|c|}{$\begin{array}{l}\Delta \text { The data of placebo pill was cited from Li, T. } 2018 \text { [27]. In this literature, the efficacy of placebo pill } \\
\text { was not associated with the baseline frequency of hot flashes, thus the corrected } E_{\max } \text { value of } \\
\text { placebo pill was consistent with the original value. }\end{array}$} \\
\hline
\end{tabular}

Based on the corrected pharmacodynamic parameters described above, the efficacy of each intervention could be estimated at different time points (Fig. 3). For example, the efficacy at week 8 of treatment for TA\&EA and SA were $3.2(95 \% \mathrm{Cl}: 2.9,3.5)$ and $2.6(95 \% \mathrm{Cl}: 2.2,3.0)$, respectively, and the efficacy of EA and TA were $3.6(95 \% \mathrm{Cl}: 3.2,4.0)$ and $3.1(95 \% \mathrm{Cl}: 2.8,3.4)$, respectively (Fig. 4).

\section{Comparison of efficacy with drug treatment}

Our previous studies showed that the efficacy of progesterone analogs, SSRIs/SNRIs, neuroleptic agents, tibolone, phytoestrogen, and placebo at week 8 of treatment were 6.4 (95\% Cl: 5.2, 7.7), 4.0 (95\% Cl: 3.5, 4.6), 3.5 (95\% Cl: 2.8, 4.2), 3.1 (95\% Cl: $2.6,3.6), 2.6$ (95\% Cl: 1.9, 3.4), and 2.3 (95\% Cl: 1.8, 2.9), respectively, after the baseline frequency of hot flashes was corrected to eight times per day. These results suggested that the efficacy of EA was comparable to SSRIs/SNRIs and neuroleptic agents, whereas TA was comparable to tibolone. SA trended to be slightly more effective than placebo pills, but the difference between them failed to reach statistical significance.

\section{Discussion}

Acupuncture is a common supplementary or alternative therapy used to relieve menopausal hot flashes [13]. However, the efficacy of acupuncture remains controversial [28]. The current meta-analysis of acupuncture for the treatment of menopausal hot flashes suggests that there was no significant difference in the efficacy between acupuncture and SA [19®21ه22ه23]. However, due to heterogeneity 
among the different studies, e.g., treatment duration, baseline frequency of hot flashes, blinding method, and other factors, the results from previous meta-analyses may be mixed with a significant amount of bias.

In this study, we found that the efficacy of acupuncture was significantly related to treatment duration. The efficacy of 8 weeks of treatment was approximately 1.6 times that of 2 weeks of treatment. In addition, we found that the efficacy of acupuncture was also related to the baseline frequency of hot flashes. For every increase in the frequency of hot flashes from baseline, the $E_{\max }$ value increased by 0.343 times/day. To determine the impact of treatment duration and baseline frequency of hot flashes on efficacy, we compared the efficacy of each intervention at the same treatment duration (8 weeks) using the same baseline level of hot flashes ( 8 times/day). The results showed that the frequency of hot flashes decreased with TA\&EA treatment at 8 weeks with the frequency being reduced by 0.6 times/day compared to that for SA treatment. However, the $95 \%$ Cls partially overlapped for these treatments due to the large variations among trials.

It is worth noting that in our current study, acupuncture intervention was divided into TA and EA, but this difference was not evident in previous meta-analyses. Our study revealed that the efficacy of EA was significantly higher than that of SA. The efficacy difference between EA and SA at 8 weeks of treatment was the reduction in the frequency of hot flashes by one per day on average. In addition, we found that the inter-individual variation for the efficacy of EA reported in the literature was smaller than that for TA and their relative standard deviations (RSD) were approximately $30 \%$ and $40 \%$, respectively [29ه30ه31]. The reason for the difference may have been due to the intensity of EA being instrument-controlled and not controlled by an acupuncture therapist. Thus, it was easier to standardize EA compared to the manual administration of TA resulting in the efficacy of EA being more stable. However, it should be pointed out that only three EA trials were included in the current study; more trials are needed to verify the efficacy of EA.

In addition to being compared with SA, we also wanted to know how effective EA was compared to existing drugs. We previously quantitatively analyzed the efficacy of different drugs in relieving hot flashes in breast cancer patients and found that the efficacies of placebo and drugs in breast cancer patients are comparable to those in natural menopausal women [27]. Our study also found that there is no significant difference in the efficacy of acupuncture in breast cancer patients and natural menopausal women. Therefore, in the current study, we directly compared the efficacy of acupuncture with the efficacies of drugs we had previously reported. The results revealed that the efficacy of EA was approximately half of that of progesterone and was comparable to SSRIs/SNRIs and neuroleptic agents such as gabapentin and escitalopram. Due to the common side effects of SSRIs/SNRIs and neuroleptic agents, such as insomnia, nausea, dry mouth, dizziness, fatigue, and anxiety, patient compliance in taking these drugs is not high [5]. Furthermore, some drugs, such as paroxetine and fluoxetine, are inhibitors of CYP2D6, which may prevent tamoxifen from being converted into its active metabolite in the body, thus affecting the efficacy of breast cancer treatments [5]. In addition, some studies have shown that acupuncture can also provide additional physical and mental health benefits such as improved sleep 
quality and reduced fatigue [1]. Therefore, EA may be a good choice for patients who are worried about drug side effects or adverse drug interactions.

Currently, SA is commonly used as a control group for clinical trials of acupuncture. However, whether placebo needles are valid controls in acupuncture research is the subject of ongoing debate. Some studies have pointed out that placebo needles may exhibit therapeutic effects, including enhanced touch sensation, direct stimulation of the somatosensory system, and activation of multiple brain systems [32]. These effects may result in physiological responses similar to real acupuncture. Therefore, in the current study, we compared the efficacy of SA with the placebo response in previous drug intervention trials. The results showed that although the efficacy of SA in reducing the frequency of hot flashes was 0.3 times/day higher than that of the placebo pills at 8 weeks of treatment, there was no statistically significant difference due to large variations among the trials. In addition, there are various forms of SA, including non-acupoint shallow thorns and non-piercing blunt needle SA. Subgroup analyses showed that the efficacies of the non-acupoint shallow thorn SA group and the non-piercing blunt needle SA group were $2.4(95 \% \mathrm{Cl}: 1.5,3.2)$ and $2.9(95 \% \mathrm{Cl}: 2.6,3.2)$ at 8 weeks, respectively. There was no significant difference between these two treatments. It should also be noted that although the efficacy of SA was not significantly better than that of placebo pills, this does not mean that acupuncture is simply a placebo. One study demonstrated that placebo needles have unique characteristics that are different from those of placebo pills [33]. Interestingly, we found that while there was no significant difference between TA\&EA and SA, the efficacy of TA\&EA was significantly higher than that of placebo pills. The difference in efficacy between TA\&EA and placebo pills at 8 weeks was 0.9 hot flashes/day. These results suggested that even though the difference in efficacy between SA and placebo pills was small, the difference was sufficient to influence the conclusion regarding whether acupuncture was effective.

The evaluation of changes in the frequency of hot flashes was relatively subjective as the daily frequency of hot flashes was self-reported by the patients [34]. Thus, the inter-individual variation of efficacy values for such trials is relatively large. Usually, this type of trial requires a large sample size to obtain a high power. However, the sample sizes of the acupuncture clinical trials included in our current analyses were generally low and most of them failed to estimate the sample sizes. Through simulations, we found that when the sample size of each arm was 200, the absolute success rate of EA or TA was better than that of SA at $74 \%$ and $62 \%$, respectively. However, if we continued to increase the sample size, there was no obvious increase in the success rate. This may be one reason for many clinical trials coming to invalid conclusions about acupuncture.

There are some notable limitations for the current study. First, there was a large difference in the baseline of hot flashes among the different studies. In four of these studies, the baseline of hot flashes was less than $5 /$ day (mild to moderate hot flashes). However, sensitivity analysis demonstrated that by removing these studies of mild to moderate hot flashes, the results were basically consistent with those of the original data set (Supplementary Table S3). This indicated that the original results were robust. In addition, the acupoints used in the various trials were different, which may have also contributed to the inconsistent conclusions among the studies. However, due to the relatively small amount of literature

Page $11 / 18$ 
included in the current analysis, it was not possible to analyze the combination of different acupoints. Finally, only published studies and those written in English were included, which may have introduced published and language biases.

\section{Conclusions}

In the current study, the efficacy of acupuncture in relieving menopausal hot flashes was quantitatively analyzed by establishing a pharmacodynamic model. We found that the efficacy of acupuncture (TA and EA) was better than that of SA and was significantly better than that of placebo pills. We also found that the efficacy of EA was more stable than that of TA, and its efficacy was significantly better than that of SA. Furthermore, the efficacy of EA was comparable to that of SSRIs/SNRIs and neuroleptic agents such as gabapentin and escitalopram.

\section{List Of Abbreviations}

Not applicable.

\section{Declarations}

Ethics approval and consent to participate:

Not applicable.

\section{Consent for publication:}

Not applicable.

\section{Availability of data and materials:}

All data generated or analysed during this study are included in this published article and its supplementary information files.

\section{Competing interests:}

The authors declare that they have no competing interests.

\section{Funding:}

This was not an industry supported study. This work was received financial support from National Major S\&T Project (2018ZX09734005, 2018ZX09711001-009-011, 2018ZX09731016, 2017ZX09304003, and 2018ZX10303501), the project of Shanghai Municipal Health Planning Commission (2018YQ48), and Shanghai S\&T Innovation Plan (17401970900). The authors declared no potential conflicts of interest with respect to the research, authorship, and/or publication of this article. 


\section{Authors' contributions:}

LJ.L and QS.Z participated in conception and design of the work and revised the paper critically for important intellectual content. T.L \& Y.Z selected studies and extracted the data, analyzed and interpreted the data, T.L \& QQ.C wrote the manuscript and revised the manuscript. MY.H and XJ.Z contributed to data extraction and cleaning. All authors read and approved the final manuscript. All authors have approved the final article.

\section{Acknowledgements:}

Not applicable.

\section{Declarations of interest:}

none.

\section{References}

1. Dodin , S., Blanchet, C., Marc, I., Ernst, E., Wu, T., Vaillancourt, C., et al. (2013). Acupuncture for menopausal hot flushes. Cochrane Database of Systematic Reviews, 7(7), CD007410.

2. Ee, C., Xue, C., Chondros, P., Myers, S. P., Pirotta, M. (2016). Acupuncture for menopausal hot flashes: a randomized trial. Annals of internal medicine, 164(3).

3. US Food and Drug Administration. SUMMARY REVIEW. Application number: 2045160rig1s000.2013. Available from: https ://www.accessdata.fda.gov/drugsatfda_docs/ nda/2013/2045160rig1s000SumR.pdf.

4. Li, L., Xu, L., Wu, J., Dong, L., Lv , Y., Zheng, Q. (2017). Quantitative analysis of placebo response and factors associated with menopausal hot flashes. Menopause, 24(8).

5. Li, L., Xu, L., Wu, J., Dong, L., Zhao, S., Zheng, Q. (2016). Comparative efficacy of nonhormonal drugs on menopausal hot flashes. European Journal of Clinical Pharmacology, 72(9), 1051-1058.

6. Biglia , N., Sgandurra, P., Peano, E., Marenco, D., Moggio, G., Bounous, V., et al. (2009). Non-hormonal treatment of hot flushes in breast cancer survivors: gabapentin vs. vitamin e. Climacteric, 12(4), 310318.

7. Pandya K, Raubertas R, Flynn P, Hynes H, Rosenbluth R, Kirshner J, et al. (2000) Oral Clonidine in Postmenopausal Patients with Breast Cancer Experiencing Tamoxifen-Induced Hot Flashes: A University of Rochester Cancer Center Community Clinical Program Study Oncology. Ann. Intern. Med, 132(10).

8. Loprinzi C, Kugler J, Sloan J, Mailliard J, LaVasseur B, Barton D, (2000). Venlafaxine in management of hot flashes in survivors of breast cancer: a randomised controlled trial. Lancet, 356(9247).

9. Wu, M. F., Hilsenbeck , S. G., Tham, Y. L., Kramer, R., Elledge, R. M., Chang, J. C., et al. (2009). The efficacy of sertraline for controlling hot flashes in women with or at high risk of developing breast 
cancer. Breast Cancer Research \& Treatment, 118(2), 369-375.

10. Nelson H, Vesco K, Haney E, Fu R, Nedrow A, Miller J, et al. (2006). Nonhormonal therapies for menopausal hot flushes: systematic review and meta-analysis. JAMA, 295(17).

11. Albertazzi P, Pansini F, Bonaccorsi G, Zanotti L, Forini E, De Aloysio D. (1998). The effect of dietary soy supplementation on hot flushes. Obstetrics \& Gynecology,91(1), 6.

12. Li, L., Lv, Y., Xu, L., Zheng, Q. (2014). Quantitative efficacy of soy isoflavones on menopausal hot flashes. British Journal of Clinical Pharmacology, 79(4), 593-604.

13. Pitkin, J. (2010). Alternative and complementary therapies for the menopause. Maturitas , 66(4), 333343.

14. Lee IS, Cheon S, Park JY. (2019). Central and Peripheral Mechanism of Acupuncture Analgesia on Visceral Pain: A Systematic Review. Evid Based Complement Alternat Med.

15. Macpherson, H., Thomas, K., Walters, S., Fitter, M. (2001). The york acupuncture safety study: prospective survey of 34000 treatments by traditional acupuncturists. BMJ, 323(7311), 486-487.

16. Deng G, Vickers A, Yeung S, D'Andrea GM, Xiao H, Heerdt AS, et al. (2007). Randomized, Controlled Trial of Acupuncture for the treatment of Hot Flashes in Breast Cancer Patients. Journal of Clinical Oncology, 25(35).

17. (2015) Nonhormonal management of menopause-associated vasomotor symptoms: 2015 position statement of The North American Menopause Society. Menopause 22(11):1155-1174.

18. Einar, B., Adrian, W. (2010). A review of acupuncture for menopausal problems. Maturitas, 66(2), 131134.

19. Chien, T. J., Hsu, C. H., Liu, C. Y., Fang, C. J. (2017). Effect of acupuncture on hot flush and menopause symptoms in breast cancer- a systematic review and meta-analysis. Plos One, 12(8), e0180918.

20. Chiu, H. Y., Pan, C. H., Shyu, Y. K., Han, B. C., Tsai, P. S. (2015). Effects of acupuncture on menopauserelated symptoms and quality of life in women in natural menopause: a meta-analysis of randomized controlled trials. Menopause,22(2), 234.

21. Chiu, H. Y., Shyu , Y. K., Chang, P. C., Tsai, P. S. (2016). Effects of acupuncture on menopause-related symptoms in breast cancer survivors: a meta-analysis of randomized controlled trials. Cancer Nursing, 39.

22. Salehi, A., Marzban, M., Zadeh, A. R. (2016). Acupuncture for treating hot flashes in breast cancer patients: an updated meta-analysis. Supportive Care in Cancer, 24(12), 4895-4899.

23. Nam, E. Y., Park, J. Y., Lee, J. Y., Jo, J., Kim, D. I. (2018). Traditional acupuncture for menopausal hot flashes: a systematic review and meta-analysis of randomized controlled trials. European Journal of Integrative Medicine, 17, 119-128.

24. Dodin, S., Blanchet, C., Marc, I., Ernst, E., Wu, T., \& Vaillancourt, C., et al. (2013). Acupuncture for menopausal hot flushes. Cochrane Database of Systematic Reviews, 7(7), CD007410. 
25. Magnusdottir, Bergrun, T.. (2016). Optimal designs for a multiresponse emax model and efficient parameter estimation. Biometrical Journal, 58(3), 518-534.

26. Vehtari, A., Gelman, A., Gabry, J.. (2015). Practical bayesian model evaluation using leave-one-out cross-validation and waic. Statistics and Computing, 27, 1-20.

27. Li, T., Yang, J., Lv , Y., Yin, F., Xu, L., Liu, H., et al. (2018). Quantitative comparison of drug efficacy in treating hot flashes in patients with breast cancer. Breast Cancer Research and Treatment, 173(3).

28. Cho SH, Whang WW. (2009). Acupuncture for vasomotor menopausal symptoms: a systematic review. Menopause, 16(5).

29. Frisk, J., Kallstrom , A., Wall, N., Hammar, M. (2009). Long term follow up of acupuncture and hormone therapy on hot flushes and well-being in women with breast cancer-a prospective, randomized multi centre trial. Cancer Research, 69(2 Supplement), 2162.

30. Nedstrand, E., Wijma, K., Wyon, Y., Hammar, M. (2005). Vasomotor symptoms decrease in women with breast cancer randomized to treatment with applied relaxation or electro-acupuncture: a preliminary study. Climacteric, 8(3), 243-250.

31. Wyon , Y., Wijma, K., Nedstrand, E., Hammar, M. (2004). A comparison of acupuncture and oral estradiol treatment of vasomotor symptoms in postmenopausal women. Climacteric, 7(2), 153-164.

32. Walker, E. M., Rodriguez, A. I., Kohn, B., Ball, R. M., Pegg , J., Pocock, J. R., et al. (2010). Acupuncture versus venlafaxine for the management of vasomotor symptoms in patients with hormone receptorpositive breast cancer: a randomized controlled trial. Journal of Clinical Oncology, 28(4), 634-640.

33. Younbyoung, C., Ye-Seul, L., Paul, E. (2018). How placebo needles differ from placebo pills? Frontiers in Psychiatry, 9, 243-.

34. Carpenter, J. S., Neal, J. G. (2005). Other complementary and alternative medicine modalities: acupuncture, magnets, reflexology, and homeopathy. American Journal of Medicine, 118(12-suppS2), 0-117.

\section{Figures}




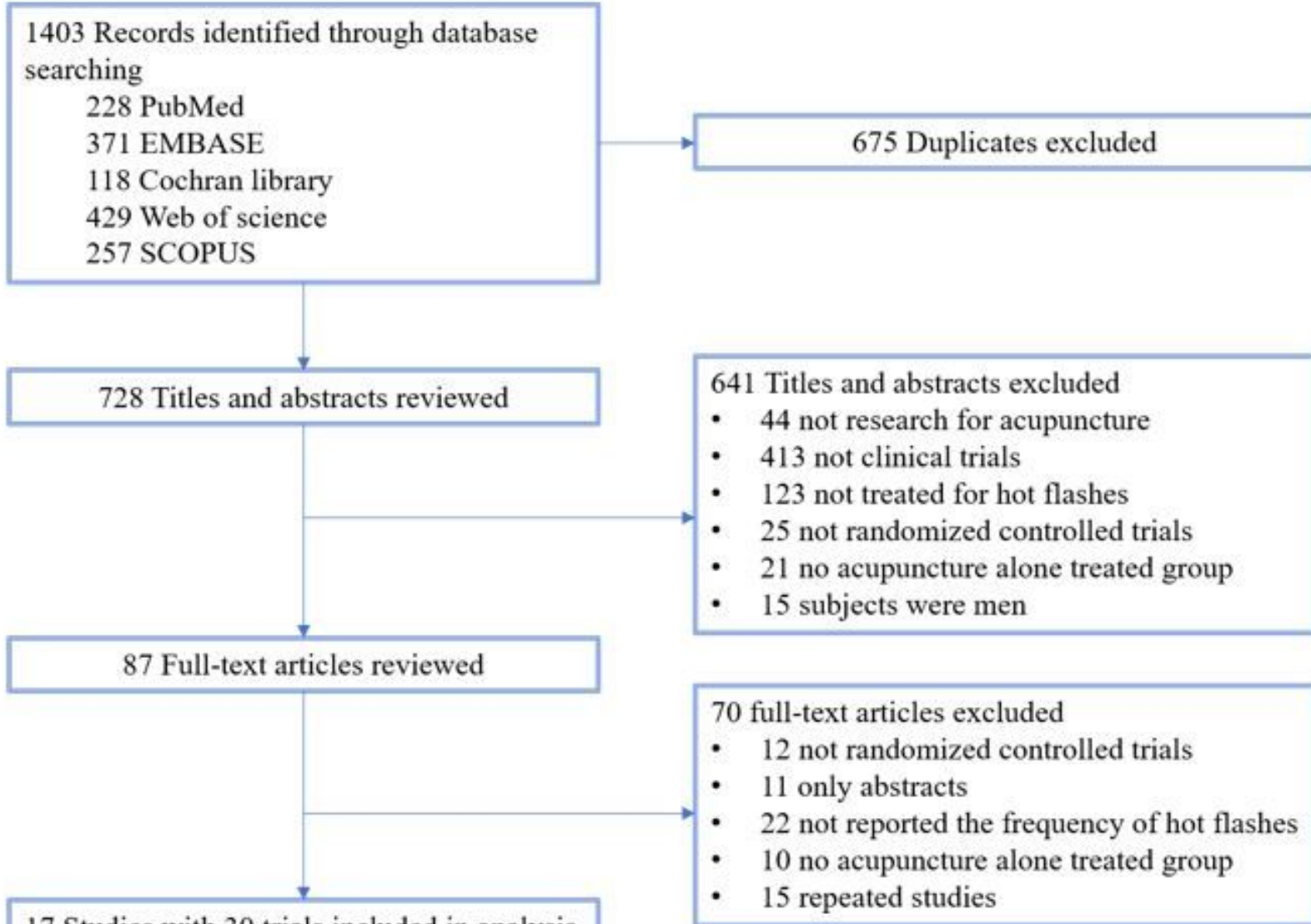

17 Studies with 30 trials included in analysis

- 13 Traditional acupuncture (TA, N=631)

- 13 Sham acupuncture (SA, N=426)

- 4 Electroacupuncture (EA, $N=69$ )

\section{Figure 1}

Flow chart demonstrating the inclusion and exclusion of studies into the analysis

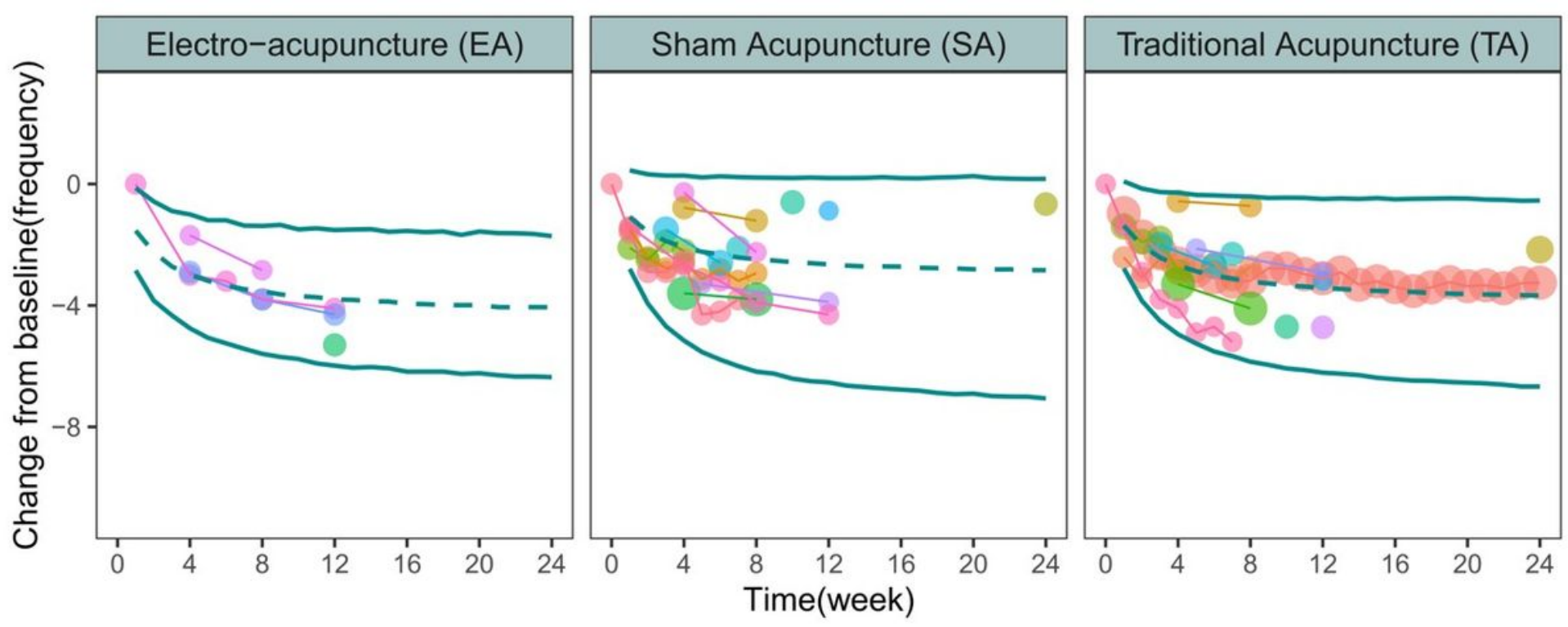


Figure 2

Visual predictive check of the final model. Solid points represent observed efficacy data, and symbol size is proportional to sample size. Points linked by a line are from the same arm. Blue lines are the modelpredicted 5th, 50th, and 95th percentiles of efficacy.

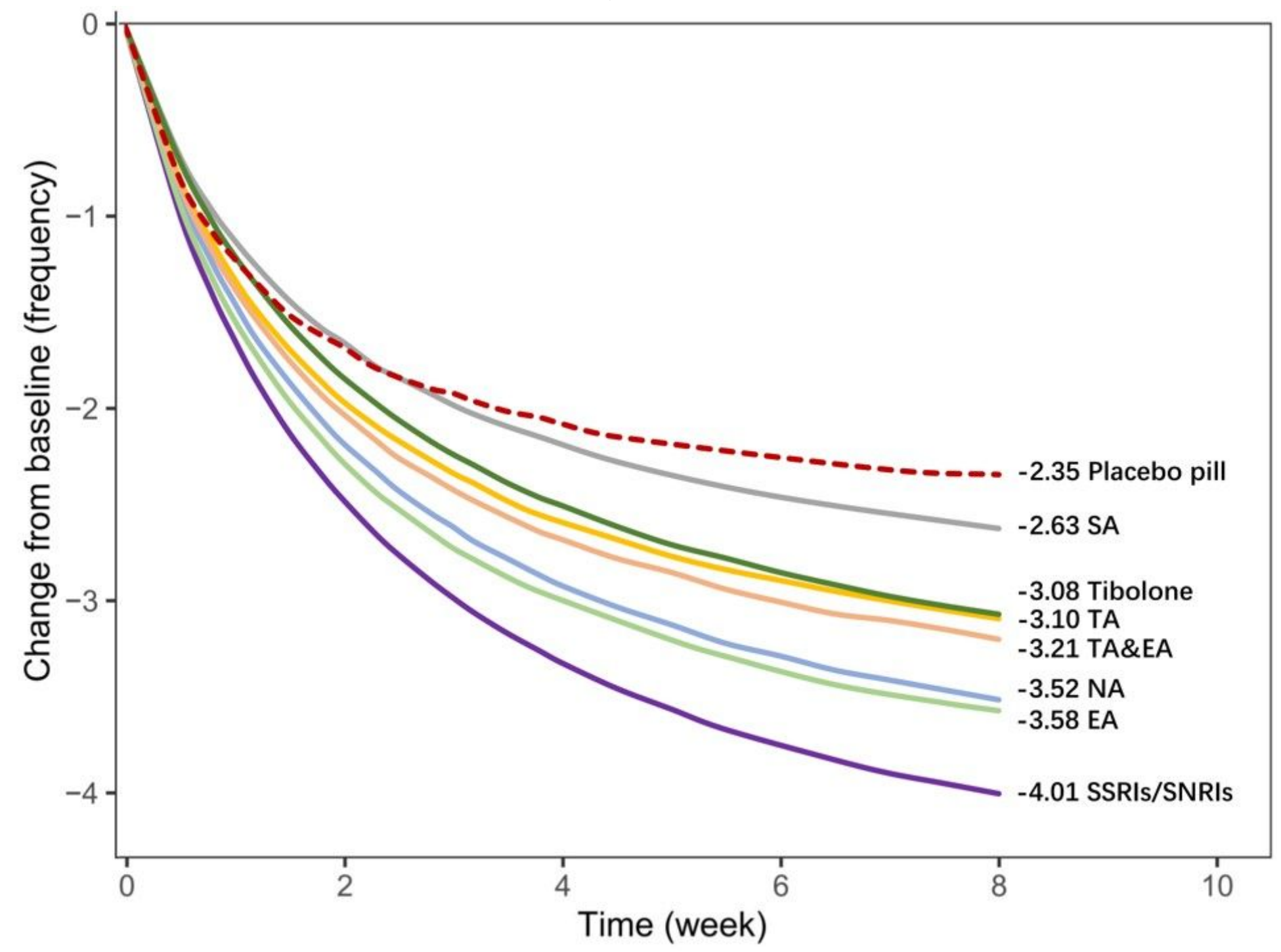

Figure 3

Predicted typical time course of hot flash reduction of each treatment. SA: sham acupuncture; TA: traditional acupuncture; NA: neuroleptic agents; EA: electro-acupuncture; TA\&EA: merger analysis of traditional acupuncture and electro-acupuncture. 

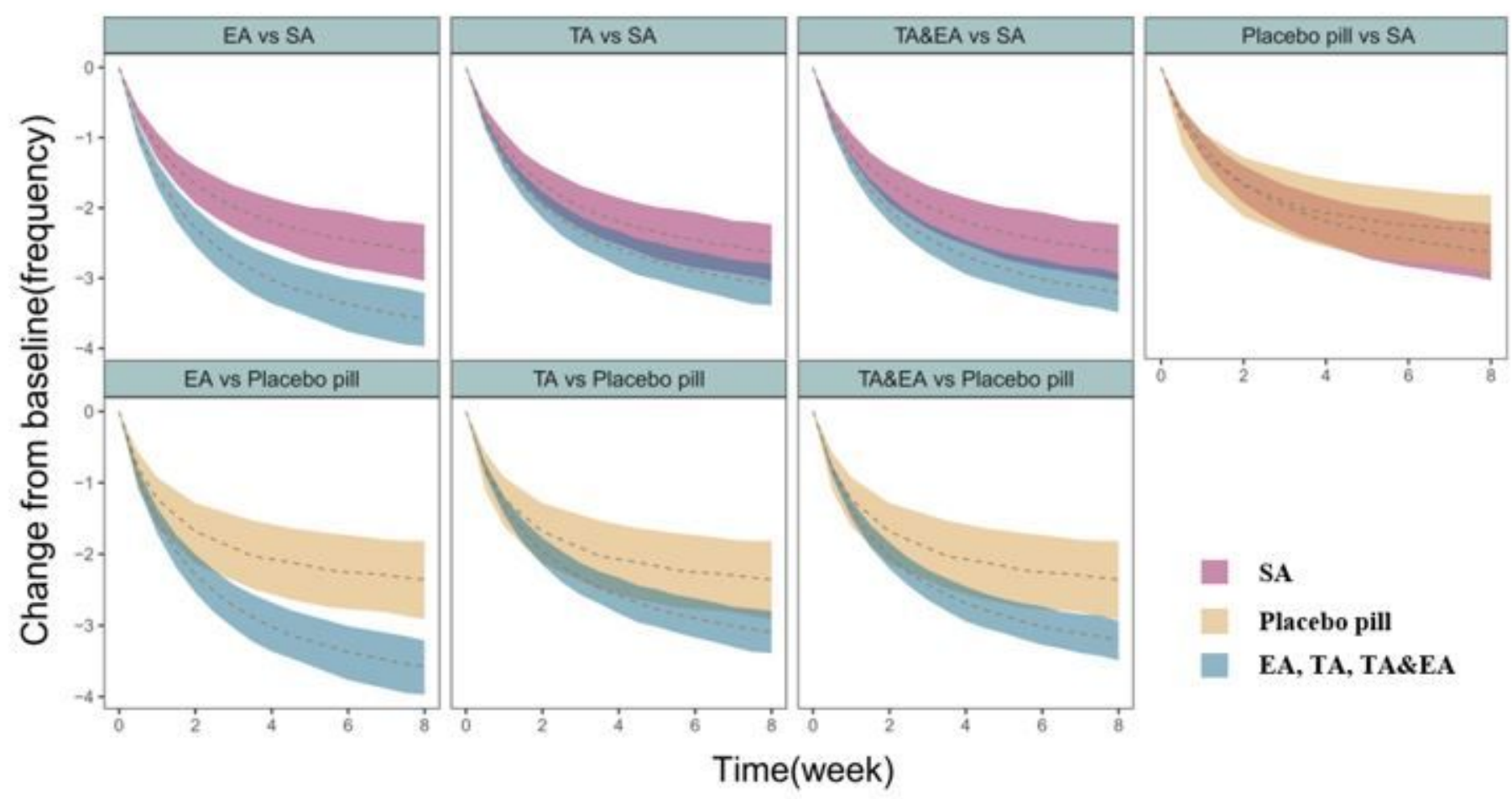

\section{Figure 4}

The predicted distribution of typical response of each treatment at the baseline level of 8 . The dotted lines represent the typical efficacy, and the shaded areas are their 95\% Cls. EA: electro-acupuncture; SA: sham acupuncture; TA: traditional acupuncture; TA\&EA: merger analysis of traditional acupuncture and electro-acupuncture.

\section{Supplementary Files}

This is a list of supplementary files associated with this preprint. Click to download.

- Supplementarymaterial.docx 